\title{
Library Pathfinders: A New Possibility for Cooperative Reference Service
}

\begin{abstract}
Library cooperation in reference services has been limited and largely unstructured. Library Pathfinders offer the opportunity to share reference methodology in an organized, nationwide manner. Intended to introduce library users to the variety of information sources available in research libraries, Pathfinders have been published in a wide range of disciplines. At M.I.T.'s Barker Engineering Library, they have proven to be effective time-savers for both library users and professional staff. The authors describe Pathfinders in detail and indicate how these reference aids can provide improved user service and instruction.
\end{abstract}

\begin{abstract}
$I_{\mathrm{s}}$ N THE CURRENT PERIOD of budget reductions that threaten the successful operation of research libraries there are signs of hope for the future in the emergence of a new emphasis on library networks and on improved interlibrary cooperation. If cooperative operations can rescue the libraries from deterioration of services that would result from budget cuts, then the time is right to study and evaluate new or improved methods of unified action that promise improved service at low cost.
\end{abstract}

Mr. Charles H. Stevens is executive director of the National Commission on $\mathrm{Li}$ braries and Information Science. Prior to January 1, 1972 he was associate director for Library Development of Project Intrex and director of the Model Library Project.

Miss Marie P. Canfield is a staff member of the Model Library Project with major responsibility for Pathfinder development.

Mr. Jeffrey J. Gardner has been a staff member of the Model Library Project since 1969 and director since January 1, 1972.

Library Pathfinders have been developed under a grant from the Council on Library Resources, Incorporated.
Although many forms of cooperation exist, a review of library activities shows that few have concentrated on reference work. In fact, no notice appears in the literature of a system for sharing the methodology for identical or similar literature search. A plan for sharing the scheme of a basic reference search has been developed within the Model Library Program of Project Intrex, Massachusetts Institute of Technology. ${ }^{*}$ The product has been named "Library Pathfinder."

\section{BACKGROUND}

Among library users are many who could benefit from professional assistance in the use of the collections. Some of these users recognize their situation and ask for the help they require; others do not; and a few do not even recognize their need. Those who do ask for assistance have questions that can be divided quite easily into four groups: di-

- The Model Library Program of Project Intrex is funded by the Council on Library Resources, Inc. 
rectional ("Where is the fiction collection?"); ready reference ("What is the capital of Mali?"); search ("What literary forgeries came to light in the 1940s?"); and library instruction ("Please show me how to gather a list of books, articles, reports, and theses on powder metallurgy.").

The direction question is generally answered from the librarian's familiarity with the surroundings. The ready reference question can usually be answered from a single source, well-known to the professional librarian. Search questions-those involving a multiplicity of media and sources plus intricate tracing of faint clues-may yield only to persistent application of intuition and intelligence. Results are seldom shared beyond the requester; only rarely will a note appear in the literature documenting a search and its results.

Questions that call for instructional answers are numerous and time-consuming for the librarian, but rewarding in the sense that, after the question is answered, the user is better able to respond to questions of the same type. However, the lasting utility of the instruction given in answer to a question requiring the librarian to serve as teacher depends on several factors. Does the librarian relate well to the requester? Is there rapport and communication? Is the question skillfully stated and does the librarian have the insight to seek the real question behind the stated query? Does the librarian have or take the time to answer the question with the fullness that satisfies but does not bore or burden the user? Is the librarian on duty the best one on the staff to answer each of the questions he or she will be asked? Can the librarian be effective on a one-to-one instructional basis when the same questions recur several times each day or each hour? If all of these questions receive satisfactory answers at all times the library cannot be faulted for its skill in instructional reference work.
These questions are tough ones and honest answers will reveal weaknesses in most libraries. Assuming weaknesses exist, is there something that can be done to help the user who needs better instructional service from the library? Library Pathfinders can provide improved service for many users.

\section{What It Is}

The Library Pathfinder is a kind of map to the resources of the library; it is an information locator for the library user whose search for recorded materials on a subject of interest is just beginning. A compact guide to the basic sources of information specific to the user's immediate needs, it is a step-bystep instructional tool that will, if followed, place before the user those items that the most skilled reference librarian would suggest as basic to an initial investigation to the topic.

\section{What IT Is Not}

Each Pathfinder carries the legend, "Library Pathfinders-designed to help users begin to locate published information in specific fields-are prepared under a grant from the Council on $\mathrm{Li}$ brary Resources. ..." An easily overlooked but important word in this statement is "begin." Pathfinders are not guides to the literature in any exhaustive sense; they are not bibliographies; they are not primarily accessions tools. Specialists may find them pedestrian and some reference librarians would deem them simplistic and perhaps trivial for their purposes; but they are not designed to serve either the experienced scholar or the reference librarian. They are for beginners who seek instruction in gathering the fundamental literature of a field new to them in every respect. Pathfinders are designed to be useful for the initial stages of library research.

\section{Pathfinder Format and Content}

The Library Pathfinder format is 
fixed but flexible. In the upper righthand corner, where it is easily seen in a notebook or a file, is the Pathfinder title. Below the title the arrangement is in two columns. Each entry includes call number and location information. Each Pathfinder begins with a "scope note," a phrase or two to delimit the topic and to insure that the user has a Pathfinder in the area of his interest. Notice of the location of beginner's introduction to the topic follows the scope note. The rationale for such an introduction is that the beginner's first need for the broad view and for an acquaintance with the terms and language of his topic. Sometimes this introduction is an entry in a standard or specialized encyclopedia; sometimes it is a book chapter or journal article. The introduction citation includes sufficient information for the user to locate the specific sources; viz., the volume and page number in an encyclopedia or journal, the entry term if the page number is not the best lead, and the chapter or range in a text. The following are examples of title, scope note, and introduction entry:

\section{GROUND WATER SEEPAGE}

SCOPE: Subsurface water movement through soil pores to the soil surface; includes ground water flow into surface water bodies and into earth structures.

An introduction to this topic appears in the McGraw-Hill Encyclopedia of Science and Technology (1971) v.12, pp.507-509 under the entry "Soil MechanicsSeepage and Frost." Q121 .M147 1971 v.12

5th Floor

Following the note on the location of an introductory article, the Pathfinder gives the user the necessary information for using the card catalog without frustration. The Pathfinder reads, "Books dealing with (topic) are listed in the subject card catalog. Look for the subjects ..." and then lists those standard Library of Congress subject headings that are in current use. This assures that the user will not unintentionally seek entries under words that are not used in the catalog and may not even appear on a "see" reference card. If entries are in more than one location in the card catalog, the user is led to each location and each subject heading is rated as either: "highly relevant," "also relevant," "related," or "more general." This distinction assists the reader who is beginning an exhaustive search as well as the one whose depth of inquiry is more limited.

In some topic areas there are texts that are mentioned again and again; these may be classic old tomes or important new books. The next section of the Pathfinder lists from one to six of these frequently mentioned texts to place their names before the user early in his work. The citations are abbreviated bibliographical entries including author, title, and date, and even page range or chapter(s) when such a limit is possible, but omitting the publisher's name, the place of publication, and any descriptive notes.

"Where to browse" information comes next. The user is given the call numbers of the stack areas that should be mined if the user prefers this method of search. In practice it seems that more than one area is usually helpful and the call numbers for each are given.

Handbooks, encyclopedias, and encyclopedic dictionaries that contain information of high utility in the subject are listed in the next section of the Pathfinder. If numerical values or tables of importance are included they are mentioned in the entry. In each case the relevant page numbers are given to speed the user's search.

At this point the order of the entries follows the pattern that we believe will be most helpful to the user of the specific Pathfinder. In science and engineer- 
ing the item that follows is a short list of bibliographies that contain citations to the literature of the topic. The Pathfinder citation to a bibliography gives the name of the bibliography, its date, the extent of coverage in number of references when this can be easily determined, and/or the pertinent page numbers. When a definitive or pertinent bibliography appears as part of a book, once again the relevant pages are given.

Bibliographies are followed by indexes and abstracting journals. The Pathfinder reads, "Journal articles and other literature on (topic) are indexed primarily in the guides listed. The quoted subject headings are those in use since 1965 unless other dates are given." Each entry includes the name of the tool, a brief indication of its coverage, and the proper entry terms to use for the topic of the Pathfinder. The following are sample entries from two Pathfinders:

TOPIC: BLACK AMERICAN

NOVEL-20TH CENTURY

Social Sciences and Humanities Index (1965+; formerly International Index) (200+ American and British scholarly journals) See:

"Negro Literature (American)"

"American Literature-Negro Authors" A13 .R2861 Humanities Library

\section{TOPIC: HEAT TRANSFER- ABSORPTIVITY}

International Aerospace Abstracts

(Covers aero-astro literature) See:

"Absorptance"

"Absorptivity"

"Thermal Absorption"

(relevant)

(also relevant)

(more general)

\section{ZTL790 .I61}

Certain topics require guides to newspaper indexes and entry words used by these indexes. When these are germane they are listed with sufficient detail to be genuinely useful.

A short listing of current journal titles of first importance follows the listing of indexes. These titles are included to put before the reader the articles in the recent literature that will not yet have appeared in the indexes. The user, with a list of most-used journal titles in the field, can scan the articles listed in recent issues and bring his search up-todate without unnecessary effort.

In some fields critical works are of singular importance and they are grouped together to assist the user. For other fields the heavily used state-of-theart reviews and conference proceedings are listed, followed by a compilation of report indexes. Each entry is as specific as possible in a real effort to meet users' needs in one package.

\section{Pathfinders in Use}

Pathfinders have been in use in the James Madison Barker Engineering Library at the Massachusetts Institute of Technology for an entire academic year and user response is enthusiastic.

The user learns of Pathfinders from the library staff, from other students and faculty, but most often from the card catalog where Pathfinder topics are entered under relevant subject headings. Each catalog card includes the legend: "Pathfinder (Topic). For literature searching use Library Pathfinder (number). Request at desk. A Library Pathfinder is designed to help a user begin to locate published information in a specific field." In the Barker Library, Pathfinders are given to users without charge.

Not all users employ the Pathfinders as they were designed to be used-moving through the sections from first to last in an orderly sequence. But in their random application, users do find that Pathfinders give them the guidance they would otherwise seek from the reference librarian. They appreciate the 
exactness of the information given, particularly the call numbers and the page numbers. They comment favorably on the specificity of the topics, on the completeness of the coverage, and on the attractiveness of a guide that is on one sheet of paper. The chief complaint is that more Pathfinders are not available. There is continuing agitation for titles to be added without delay and the library staff is as eager as the users to satisfy this requirement.

\section{The Cooperative Program}

From the beginning it was recognized that one library could not produce a fraction of the Pathfinders it would like to offer to users. It was also obvious that the Pathfinders would be valuable both as a beginner's guide for serious investigation and secondarily as an acquisitions guide for professional personnel. In order to increase the number of Pathfinders and make them available to a larger number of library users, a cooperative program has been developed.

The first step was taken as we found a way to make the work done at M.I.T. available to others. This was accomplished by inserting an intermediate step in the final stages of Pathfinder preparation. As the draft of the final Pathfinder is typed, the library name, the call numbers and the collection locations are omitted from the text. This provides a Pathfinder that is suited to its topic but not specific to a particular library. When this Pathfinder is reproduced two sheets are used. At this stage the Pathfinder is no more ready for use at M.I.T. than it is elsewhere. One set of the reproduced sheets (i.e., the Pathfinder master) is completed by adding the name of the library (Barker Engineering Library in our case), the call numbers for each of the sources cited and the locations within the library. Then the completed Pathfinder master, adapted for M.I.T., is ready for final multiple printing-this time on two sides of one sheet of card stock drilled for a student notebook. A less expensive alternative is to file the two-page completed master for on-demand duplication on the library's copy machine at either the user's or the library's expense. Other copies of the two-page master are ready to send to other libraries that want them and have agreed to participate in the cooperative program. Participation, in this case, does not mean simply the use of the Pathfinders available from M.I.T., but includes the creation of new Pathfinder titles using the style and guidelines that have been developed for them. In this way, the library that wishes to use Pathfinders adds to the number of titles available and contributes to the shared reference activity promised in the Introduction.

Questions on many points arise all at once. What does it take to compile a Pathfinder? Who can do it? How are topics selected? How are Pathfinders prepared for initial typing? Where and how is the initial typing and printing done? What is Pathfinder exchange basis?

Compilation of a Pathfinder is a substantive task for a trained librarian. It requires about fifteen hours of effort to compile one topic. This time decreases when several related topics are done at once.

The initial task is that of topic selection. The objective has been to seek high utility and current parlance in the topics since Pathfinders that would help the most people would, at the same time, provide the best test and be the most help to the library's reference staff. Current terminology has been used to obtain the highest degree of user acceptance. The initial method for selecting topics involved a review of the noun phrases within the course descriptions in the M.I.T. Catalogue. These noun phrases were compared with those gathered from recently completed theses 
and from theses in progress. Ideas that appeared repeatedly were considered candidates for Pathfinders and a quick check with an appropriate faculty member confirmed or rejected the choices. As selections were made, consideration was also given to the collection that would be used to compile the Pathfinders. Efforts were made to avoid topics that were either too broad to be of genuine interest to users or too narrow to be helpful to more than a handful of patrons. After the appearance of the first titles, users began to ask for new titles at a rate that outstripped the ability of the Model Library staff to produce them. Requests are weighed carefully and those that meet the established criteria are selected. As cooperating libraries join the program they are urged to compile Pathfinders in areas in which their collections are strong and in which there is a demonstrated need for the product. The function of the Model Library staff, at this point, is to provide a central registry of topics to reduce the possibility of duplication of effort.

Those who compiled the first Pathfinders here at M.I.T. are the authors of this article; each is a professionally trained and experienced librarian. The staff's judgment was that this background would be essential to a finished product. Nothing has occurred to change that view. Professors and graduate students are aware of some kinds of source materials but either they do not have the full scope of the literature at their command or they do not exhibit the interest necessary to compile a Pathfinder for beginners.

However, one group of willing contributors who are qualified to perform the initial spadework on Pathfinders are students in the graduate schools of library science. Beginning with the students in Professor James Matarazzo's class in the literature of science and technology at Simmons College, programs have been initiated in a number of library schools in which Pathfinders are compiled as course assignments. The majority of the humanities and social science Pathfinders began as student assignments since only a few were done at M.I.T. as a demonstration.

The student work is incomplete and unacceptable in some cases, but better than half are useful beginnings for finished products. A Pathfinder of good quality from a student may need only five hours of work by the Model Library staff to make it ready for publication. To insure that work is not duplicated, the same care in selection of topics is exercised for school assignments as for those done by professionals. The name of the compiler and his institution appear in an acknowledgment on the verso of the final, edited Pathfinder.

To facilitate Pathfinder preparation, detailed guidelines are provided to direct the compiler towards producing a Pathfinder of standard, high quality. In addition, a worksheet is provided which has the standard introductory phrases for each section and space for entries. It is this completed worksheet that is often received from cooperating libraries.

Using masters preprinted with logo and copyright information, the typist prepares each Pathfinder for final printing. Typing time is approximately thirty minutes and each Pathfinder is, of course, proofread and corrected.

Two major areas of concern are the problems of updating Pathfinders and increasing their distribution. Pathfinders will be updated annually in areas of emerging interest, such as environmental pollution and transportation science. Titles within the more traditional subject areas, such as heat transfer and fluid mechanics, are scheduled for biennial revision.

An attractive feature of the Pathfinders, however, is their built-in updating capability. By including Library of Congress subject headings and abstracting and indexing service headings, the 
user has the capability to update the results of a Pathfinder search.

The distribution procedure has been a more serious problem. Many institutions have indicated a preference for purchasing Pathfinders over committing a significant amount of professional staff time to Pathfinder compilation. This fact, combined with our lack of ready capability to market and distribute Pathfinders on a wide scale, led to negotiations with commercial publishers and ultimately to contractual agreement with the Addison-Wesley Publishing Company which began distributing Pathfinders at a cost of $\$ 1.00$ per title.
The charge includes full internal reproduction rights and two catalog cards for entry into subject card catalogs. Editorial responsibility remains with the Model Library Program.

As the program expands there is a possibility that Pathfinders will be compiled for users in many types of libraries, including high school libraries, community college libraries, and public libraries of all sizes. As long as library users are bewildered when faced with specific information needs, Pathfinders could continue to lead them into, and perhaps through, the labyrinth. 